International Journal of Distributed and Parallel Systems (IJDPS) Vol.3, No.4, July 2012

\title{
APPLICATION OF WIRELESS NANO SENSOR NETWORKS FOR WILD LIVES
}

\author{
Vimal Upadhayay $^{1}$ and Dr. Sonali Agarwal $^{2}$ \\ ${ }^{1}$ Research Scholar, Department of Information Technology, Indian Institute of \\ Information Technology, Allahabad, Uttar Pradesh, India \\ rs93eiita.ac.in \\ ${ }^{2}$ Assistant Professor, Department of Information Technology, Indian Institute of \\ Information Technology, Allahabad, Uttar Pradesh, India \\ sonali@iita.ac.in
}

\begin{abstract}
A Wireless Nano Sensor Network (WNSN) is a collection of Nano Sensor nodes that dynamically self organize them in a wireless network without a need but with possible utilization of any pre-existing infrastructure. WNSNs may be employed on the wildlife health and condition monitoring. Most of the current research in WNSNs on wildlife monitoring is performed on theoretical basis involving simulations of abstract, unrealistic situations. The research focusing on the real life evaluation of the WNSN techniques involves usually nano-scale studies with the very limited number of nodes attached with the body of the wild animals. The major problem with the Sensors/Radio Collar which are attached to the body of wild animals is size of the mote. For which reasons they feel uncomfortable and when they try to be free from it, the mechanism would be damaged. Then it is needed to be changed and it affects the cost. And for the macro size of the Sensors/ Radio Collar, the power consumption is also high which is the main problem is for the maintainer. It requires changing the battery frequently. Finding the location of that certain animal and putting it into a cage after tranquilization claimed a huge amount time and labour.
\end{abstract}

\section{KEYWORDS}

Wireless Network, Nano-Sensors, Wildlife Movement and Health Monitoring, Low Power Consumption

\section{INTRODUCTION}

The important challenges in the proposed system are maximizing uptime of the Nano Sensor nodes and minimize its size so that the animals do not feel uncomfortable when it will be attached with its body while preserving the minimal required performance of their communicational tasks. Also comes under consideration that the Nano-scaling of sensors consume less power which clearly indicates that the monitoring of the wild animals would be continued for a certain long period without maintenance interruptions. The Wireless Sensor nodes embedded to the body of animals send physical data to the sink node device from where data would be communicated to the server at Ranger Offices. That data will be processed through data warehouse techniques for the perspective knowledge discovery. Clusters are formed such that all nodes in two adjacent clusters can communicate with each other. A cluster is composed of sensors with the same cluster ID. Periodically, each cluster elects a clusterleader. Each node may probabilistically elect itself to be the cluster leader by broadcasting a "leadership intention" packet. After the packet is received and acknowledged by other nodes in the same cluster, new head starts to role and round. It takes several steps for sensors to organize themselves into local clusters. First, sensors are densely deployed in a randomly manner in a large region. One additional sensor, carrying the information of the approximate length and width of the field area, is deployed in the centre. It is called seed node. Based on the 
International Journal of Distributed and Parallel Systems (IJDPS) Vol.3, No.4, July 2012

transmission range of the sensors, the seed node is able to draw up a grid that covers the whole sensor field. The seed node floods the network with a reference packet containing its own location information which is used as a reference point. Upon receiving the reference packet, each node compares its own location with the location in the packet, and calculates its own cluster ID. Note that the cluster establishment is a one-time only process.

\section{Previous techniques}

In the previous research the Sink node is located in the centre of the network. Started from the seed sensor, the Sink node is growing outwardly until it reaches the mid-way between the centre and the boundary. An analogy of this is the ripples. Dropping a rock into a pool, you see rings of ripples. Initially the seed broadcasts messages asking its neighbouring nodes to form a ring [2]. After this is done, nodes in the ring broadcast messages asking its outward neighbours to form a new ring. The seed sensor node is not necessarily to be deployed in the centre of the network. For certain deployment environments when the centre is not reachable, the seed sensor can be placed on the boundary of the field. However, this requires different formation scheme. After a sensor discovers an event, it starts collecting data and sending reports to sink node. The complete path to sink node is not stored in the sensors since it can be costly and the path is prone to constant changes. A sensor only saves the information of its neighbours who are onestep above and below it on the path. This information is available after Path Establishment Phase described in the previous section. After receiving the data from its neighbour, the sensor searches its own memory and forwards the data to its neighbour on the path toward Sink node. Since all the nodes on the path have only local information about the path, when any nodes are rotated out as cluster-leader or need to power themselves off due to low battery, they only need to turn over the information of its two neighbouring nodes to its replacement in order to reestablish the connections [11].

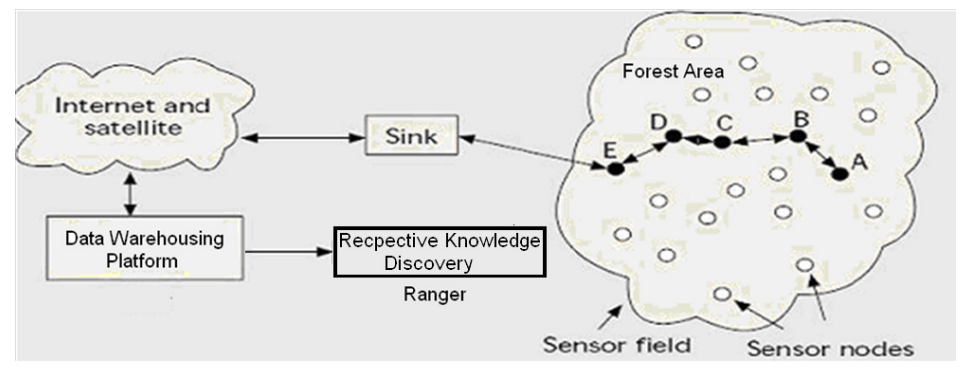

Figure 1. Traditional Sink - WSN data mining connectivity

It takes a limited number of transmission hops before the data reaches a Sink node. That Sink node then broadcasts the event information across the whole Sink node. It first sends the data to its right-hand neighbour. The recipient node checks if it had received it before [6]. If not, it saves the data and forwards it to its right-hand neighbour until the data packet is returned to the original sender. Otherwise, the data packet is considered obsolete and is dropped. If one of the Sink nodes dies, Sink node will still be connected but not in a ring shape [3]. The data packet will not be circulated and returned to its original sender. A timestamp is needed to help determine whether the packet is considered lost and should be sent in the other direction [8]. The sensed data is processed by the data mining technique and compared with respect to the standard functions and policies defined as wildlife characteristics and behaviour to gain uncertain knowledge about the physical conditions and problems of the animals.

Associations also identify groups of particular features that appear frequently closer to each other. It is called mining of spatial-co-locations [7]. Spatial Clustering is a process of grouping 
International Journal of Distributed and Parallel Systems (IJDPS) Vol.3, No.4, July 2012

spatial objects to clusters. This process ensures that the spatial objects clustered together have a similarity and dissimilarity to the objects in other clusters. This technique is applicable to a group of similar objects also all classical clustering algorithms can be used to cluster the spatial data. Spatial Classification analyzes special objects to derive classification scheme such as neighbourhood [1]. This requires the identification of spatial related factors and by performing and by performing on max properties, relevance analysis, best attributes can be selected. Then traditional classification algorithms like decision trees can be used to classify the medical data of the animal. Spatial trend analysis also can be applied to detect changes and trends along spatial dimension. This extracts trend of Spatial or non spatial data changing with space [9]. Sometimes, both time and space change. Traffic flows is an example. Spatio-temporal classification scheme can be for these sorts of data.

\section{THE STRUCTURE OF NANO SENSORS MOUNTED ON THE BODY OF} ANIMALS

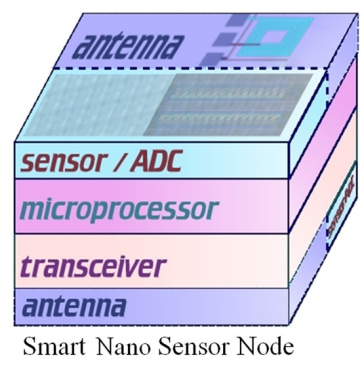

Figure 2. Diagram of Solid Structure of Nano Sensor Mote

The above figure [5] illustrates the structure of a single sensor node containing one or more MEMS/optical nano--sensors, an analog to digital interface, a processor for interpreting the nano--sensor data and controlling the network, and then a transceiver to share and receive the nano--sensor information with the outside world. The illustrated figure of the ad-hoc is based on nano--sensor network. While the long term goals are to develop sophisticated ad-hoc wireless smart nano--sensor networks that may include new nano--sensor modalities that are the results of multidisciplinary technologies such as MEMs/IR, the short term goals are to develop the electronics necessary to control the MEMS and optical nano--sensor devices, extract the sensed information, and where appropriate transform it into digital signals for micro-processing. In the long-term however [10], it is proposed to implement on-nano--sensor signal processing capabilities that communicates intelligent and relevant information rather than simple images and nano--sensory data.

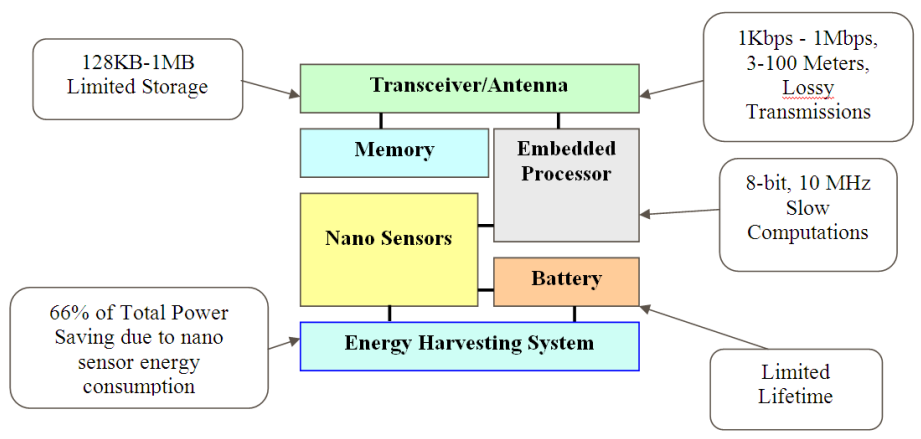

Figure 3. Block diagram of nano sensor mote with high battery backup 
Due to small energy consumption by the nano sensors, processor, antenna and memory, the duration of battery embedded to the nano sensor mote is much longer than normal sensor node which used to be deployed to the body of animal previously. For the small size of sensor node the possibility of damaging of mote is reduced sharply since the animals do not feel uncomfortable wherever it will be deployed on their body.

\section{The NANo-Sensor TeChNiQues AND Details}

The electrolyte channel length is $70 \mathrm{um}$ and electrode gap is $3 \mathrm{um}$, respectively. The potential responses of cathode during electro deposition of a (led) Pd wire at $-1000 \mathrm{nA}$ applied current does not require any electrolyte channel. Initially the cathode potential approaches a negative value, and then it gradually increases at the potential level of led wire growing from cathode to anode. When the wire is fully grown and contact to the anode, the potential drops to zero and it's turned off [9]. The 7 um long led wires were grown at $-1000 \mathrm{nA}$ within 1500 seconds. The changes in electrical resistance between gold electrodes during led wire growth at $-1000 \mathrm{nA}$. The electrical resistance gradually decreases as the led wire reduces the gap between cathodes to anode. When the led wire contacts the anode the measured resistance is less than $100 \mathrm{Ohm}$ in the liquid electrolyte. The Optical images of the nano sensor containing the electrochemical led wires between gold electrodes are shown in Figure below:

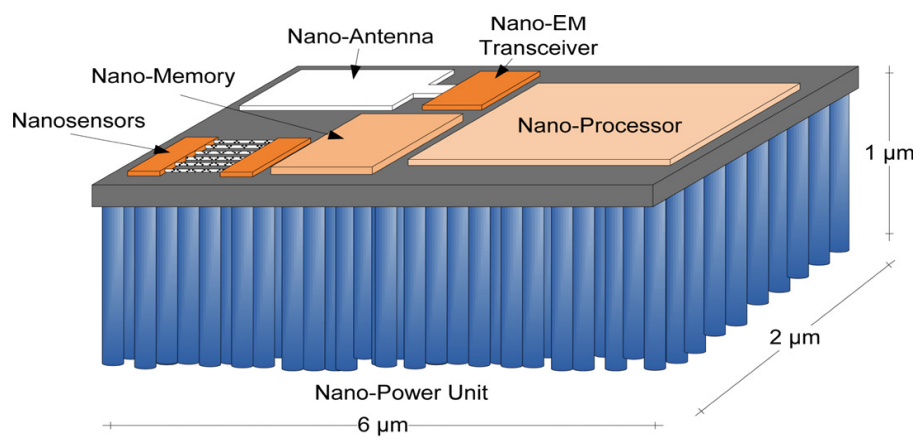

Figure 4. Arrays of Electrode with e-beam oriented electrolyte channels

By applying silicon nano-wires gowned chemically as an etch masks, the nano-walls into thin films of silicon is stenciled through electronic transport effects. This similar lithographic method can also be applied to create any patterned nano-structures of other materials besides $\mathrm{Si}$ like graphine. Under certain conditions, a periodical process nano-wire deployment can be obtained by producing a group of nano-wires stacked vertically from a single nano-wire mask. Together, these techniques highlight the potential of this nano sensing process through nextgeneration nano--electronics, sensing, and electromechanical systems [7].

After Sink node is formed, Sink node cluster leaders enter Path Establishment Phase in which they attempt to establish paths to all non-Sink node cluster leaders. First, they broadcast a "build path" packet with hop counter set to zero $\left\langle n u m_{-}\right.$of _ hops_towards_artery $\left.=0\right\rangle$. On receiving the packet, each node increments the hop count by one and compares it to its own counter $\left\langle\right.$ num of $_{-}$hops $s_{-}$towards_artery $\rangle$. If its own counter is smaller than the hop counter in the packet, it discards the packet. If it is larger than the one encoded in the packet, it has found a shorter path to sink node. It then updates its own counter and sets its next_hop pointer to the neighbour from which the packet was sent [6]. After the Path Establishment Phase, every node in the network has path gradients toward Sink node. 


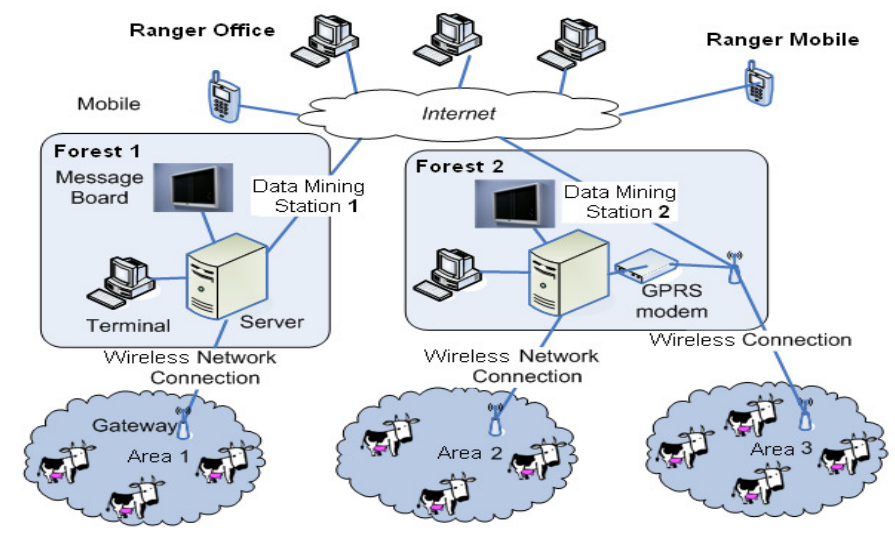

Figure 5. Prototype Deployment

Each sink node placed in various places in the forest is connected to the central server of data storage for further processing through wireless connectivity. When a sink collects data from the sensor field, it first broadcasts a query request within the cluster where it resides. Since the leader of the cluster is always awake, it picks up the query and forwards it toward Sink node in the same way as event information is forwarded. When the query reaches Sink node, the Sink node checks its memory to find if there is a match of event information. If so, it sends the event data back to the sink along the reverse path. If there is no data found, the Sink node broadcasts the query in Sink node in the same way as an event is broadcasted [10].

\section{IMPLEMENTATION OF DATa Mining INTO WILDlife Monitoring}

Since only the cluster leader is active during one round, all other non-cluster-leader nodes can be powered off, thus saving considerable amount of energy. Besides, all the data generated within a cluster can be aggregated by the cluster leader, greatly reducing the amount of outbound data. The body sensor device on the animal sends the data of physical characteristics of animal to the Forest server at Ranger office where the data could be taken for relation mining.

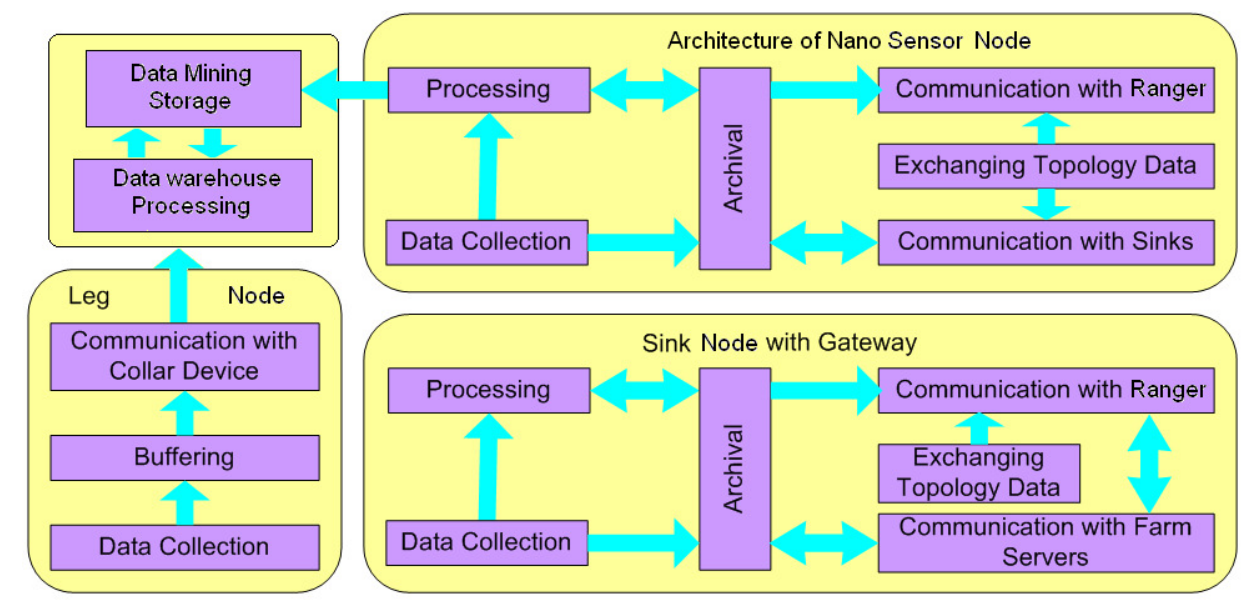

Figure 7. Animal mounted nano sensor node-functional diagram

Association rules can be applied to spatial data. The extracted association's rules are of the form $\mathrm{A} \Rightarrow \mathrm{B}(\mathrm{SY}, \mathrm{CY})$ where A \& B are sets of Spatial or non spatial predicates. SY is the support of 
the rule and CY is the confidence of the rule. Spatial associations occur at different level. For example, the following is an association rule [9].

Is_a(x, 'school') ${ }^{\wedge}$ closed_to(x, 'Big playground') => close_to(x, “city”) $(50 \%, 50 \%)$

From what is described above, we can see that Sink nodes take a much heavier load of responsibilities than non-Sink nodes thus consuming a lot more energy. If Sink node is fixed and all nodes have the same amount of battery power, the Sink nodes will die much earlier than non-Sink nodes. The network will then be partitioned into two, an inner and outer part, disconnected from each other. To solve this problem, we propose a slightly different design - a structure we call Floating Sink node. When the energy level of a Sink node is lower than certain threshold, the node transfers its duties to one of its non-Sink node neighbouring node. First, it contacts its two non-Sink node neighbours to see if their energy levels allow them to take over the job. If not, which means the neighbours have less energy, the Sink node has no choice but to remain on the job [8]. If one of its neighbours is capable of the duty, the Sink node turns over all the information it maintains, including its left and right neighbours in Sink node, all its non-Sink nodes connections, and all the event data and queries it keeps in the memory. Then the new Sink node establishes the connections and all the old paths are resumed. The whole handover process uses only local information.

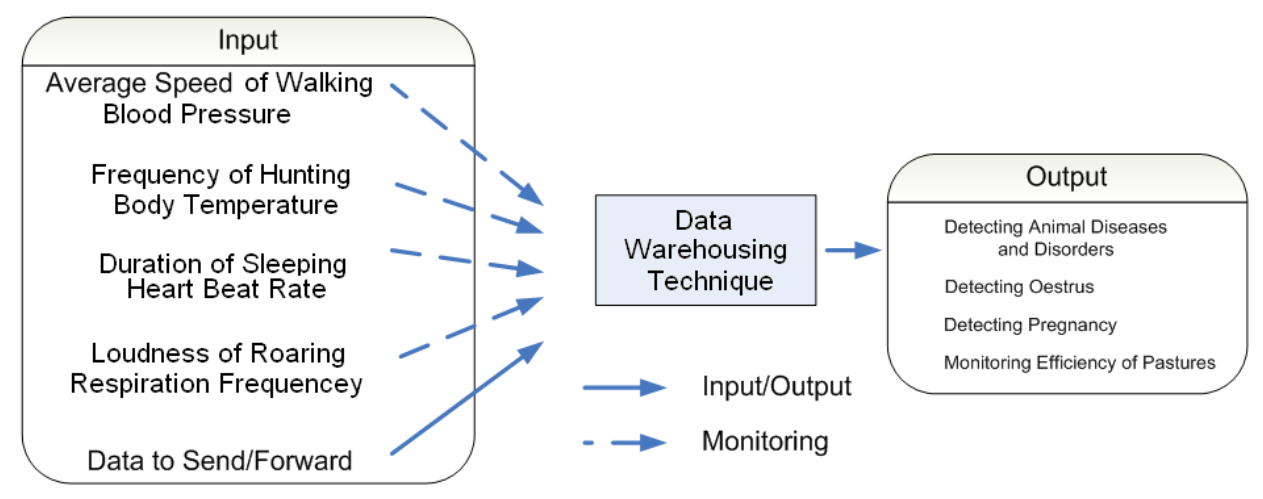

Figure 8. The Relevant Mapping through Data Warehousing Technique mounted nano sensor

The above data taken from the nano sensors mounted on body of animal represents the above factors for calculating the physical conditions of the animal through data mining technique. Information retrieval indicates that based on a query, the information can be retrieved. One good example is web search engine. Here, based on the query, the search engine performs a matching of key word with bulk of texts to retrieve user requested information. One popular method is vector-space model. In this method both document and a query represent vectors in the highdimensional space of all possible keywords. Therefore similarity measure is used to approximate document vector and query vector. Here similarity values are used to rank the documents [11].

\section{Simulated Result in QualNet AND WEKA}

The proposed wireless nano sensor network has been employed on QualNet software tool to simulate the tentative outcome from the architecture of wildlife monitoring system. The sequential numbers are the WSN nodes attached to the body of wild animal and they are connected to the central server at ranger office through sink node. 
For the proposed wireless sensor network based data mining technique for monitoring the activities of wildlife is deployed on the dataset of critical behaviour of wild animals taken from the "World Wide Fund for Nature" organization, link http://www.worldwildlife.org/science/data/WWFBinaryitem6602.zip through WEKA simulator. This technique will help to find out the relationship among the parameters of activities of wild animals during different situation like hunting, sleeping, wounded etc. The data mining technique will help to discover any unexpected knowledge about the behaviour of wild animal if any uncertain condition will arise. For an example wounds in leg of any animal can be detected by the walking speed data taken through the inertia sensor attached to its legs. So, the finding the patterns among the activities and behaviour of the wild animal with respect to their physical situation and conditions if occurs, is taken as the primary concern in this research. In the taken dataset of critical behavioural activity of wildlife only the following parameters have been focused shown as column wise:

- 7 th column : average walking speed of the animal

- 13th column : ECG rate or heartbeat of the animal

- 23 rd column : blood pressure of the animal

These above parameters are mainly responsible for detect any conditions of wild animals so that those parameters are taken for contracting the patterns among the behavioural situation of wild animal through data mining technique. The respective graph is shown below:

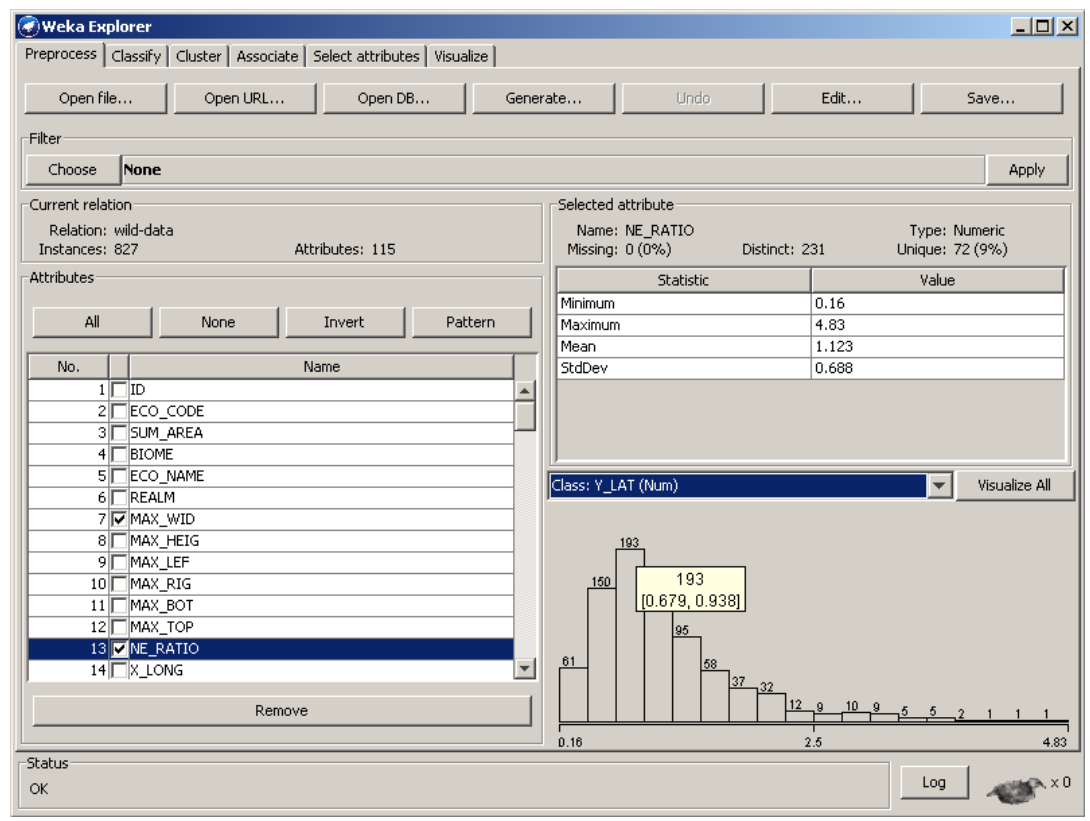

Figure 12. Bar chart of ECG rate and inertia

In the given image number 193 is the highest value of occurrence of lowest rate of ECG with respect to inertia data and blood pressure measurement which are shown in the above graph at 0.679 and 0.938 scale respectively. The condition of occurrence of such values are only happed when the animal are in sleeping situation. The relationship among the inertia and blood pressure with respect to ECG is shown below in a graphical form to detect the activity of it. 


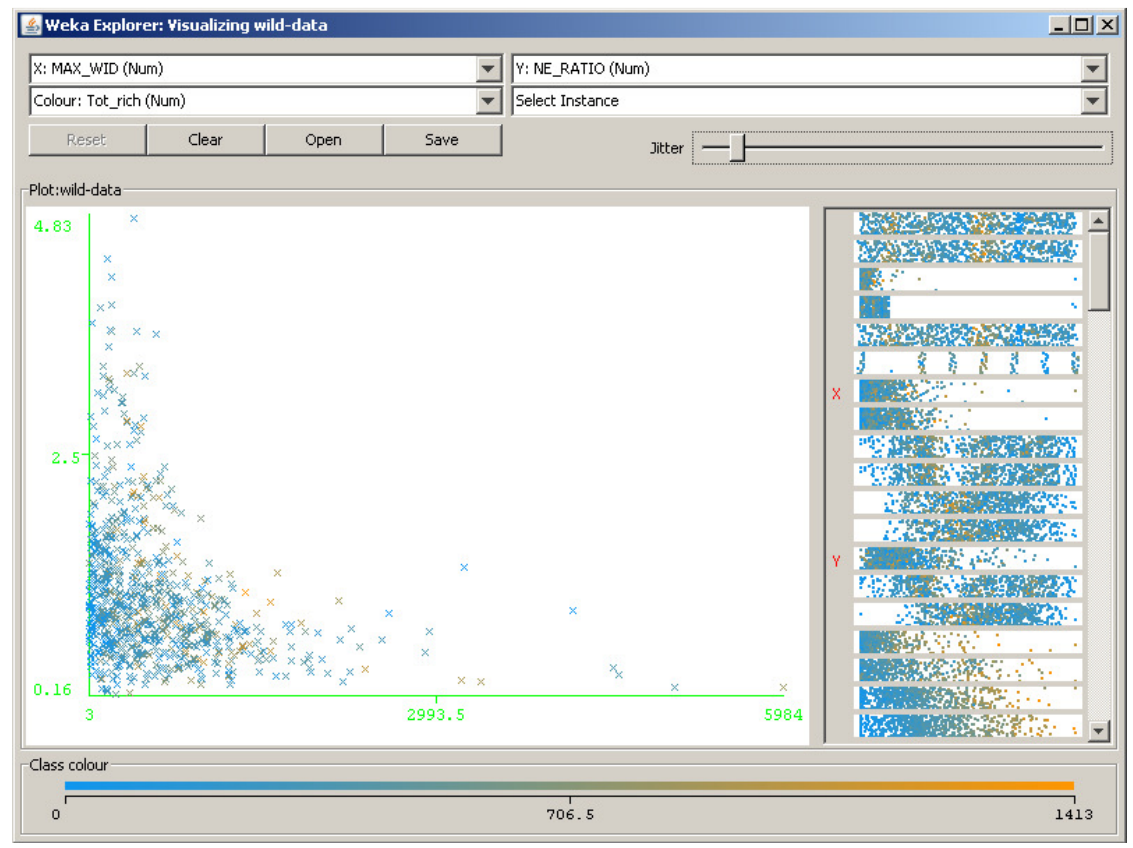

Figure 13. Graphical representation between ECG rate and blood pressure with respect to inertia

In the above image $\mathrm{X}$ axis represents the inertia value of wild animal and the $\mathrm{Y}$ axis represent blood pressure measurement with respect to the ECG rate in colour at the bottom of the graph. From the above image it is indicated that the inertia, ECG rate as well as blood pressure are very closely to each other at origin position where all the value are very low. So, it has been clearly concluded from observation of the simulated graph that the animals are resting maximum number of hours in a day.

\section{Conclusions}

AWSNs impose new challenges on the design of IDS, which are especially needed due to the unattended operations in open environments of wild animal husbandry. The network owner cannot simply rely on usual security mechanisms to ensure its security. We propose to implement a flexible and efficient intrusion detection system, which can then be used in a variety of wireless network and devices, and easily adapted to the resources available to discover the uncertain knowledge about the physical condition of animals by processing the raw data through data mining technique.

\section{ACKNOWLEDGEMENTS}

We thank to our Honourable Director Sir for allowing us to perform this research work by providing excellent academic facility under his privilege guidance.

\section{REFERENCES}

[1] Akyildiz, W. Su, Y. Sankarasubramaniam, and E. Cayirci,"A survey on Sensor Networks," IEEE Communications Magazine, vol. 40, Issue: 8, pp. 102-114, August 2002.

[2] C. Intanagonwiwat, R. Govindan, D. Estrin, J. Heidemann, and F. Silva, "Directed Diffusion for Wireless Sensor Networking," IEEE/ACM Transactions on Networking, vol. 11, pp. 2-16, Feb. 2003. 
International Journal of Distributed and Parallel Systems (IJDPS) Vol.3, No.4, July 2012

[3] W. Heinzelman, A. Chandrakasan, and H. Balakrishnan, "Energy-Efficient Communication Protocol for Wireless Micro-Sensor Networks," in Proc. of the 33rd Annual Hawaii International Conf. on System Sciences, pp. 3005- 3014, 2000

[4] P. Boonma, P. Champrasert and J. Suzuki, "BiSNET: A Biologically-Inspired Architecture for Wireless Sensor Networks," In Proc. of the 2nd IEEE/IARIA International Conference on Autonomic and Autonomous Systems (IEEE/IARIA ICAS), Santa Clara, CA, July 2006

[5] Prof. Martin Peckerar, Prof. Neil Goldsman, Mr. Luke Currano, Dr. Nibir Dhar and Dr. Madan Dubey, "Hardware Design for Realization of Smart Sensor Network A Proposal to the Army Research Laboratory PEER Program”, Dept. of Electrical and Computer Engineering, University of Maryland

[6] Lionel Besson, Philippe Leleu, "A distributed intrusion detection system for ad-hoc wireless sensor networks”, IEEE publication 978-1-4244-4530-1/09.

[7] Lan LIN, Hailin WU, "Artery: A Data-Centric Architecture for Wireless Sensor Networks", journal of Systemics, Cybernetics And Informatics, Volume 1 - Number 4, page 78 - 83.

[8] Lionel Besson, Philippe Leleu, "Developing a Cooperative Intrusion Detection System for Wireless Sensor Networks", NATO-OTAN, RTO-MP-IST-091, page 21-1 to 21-8.

[9] Dr Milena Radenkovic and Prof. Chris Greenhalgh, "Application of Mobile Ad-Hoc Networks to Monitoring of Farm Animals", Technical Overview.

[10] Bartosz Wietrzyk, Milena Radenkovic, "Realistic Large Scale ad hoc Animal Monitoring", School of Computer Science, University of Nottingham.

[11] Shah Sheetal, “Autonomic Wireless Sensor Networks", Viterbi School of Engineering, University of Southern California, Los Angeles, CA, USA.

\section{Authors}

Mr. Vimal Upadhaya, Research Scholar

Indian Institute of Information Technology, Allahabad, India.

Dr. Sonali Agarwal, Assistance Professor

Indian Institute of Information Technology, Allahabad, India
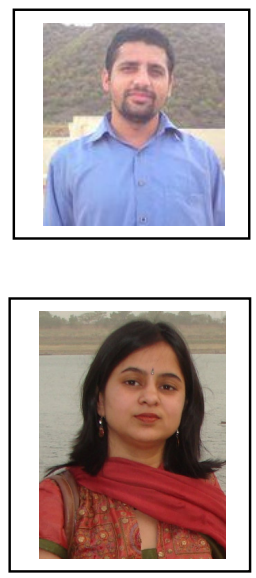